\title{
COX5A over-expression protects
} cortical neurons from hypoxic ischemic injury in neonatal rats associated with TPI up-regulation

Ya Jiang ${ }^{1 \dagger}$, Xue Bai ${ }^{3 \dagger}$, Ting-Ting Li ${ }^{3 \dagger}$, Mohammed AL-Hawwas ${ }^{2}$, Yuan Jin' ${ }^{1}$, Yu Zou' ${ }^{1}$, Yue Hu ${ }^{1}$, Lin-Yi Liu ${ }^{4}$, Ying Zhang ${ }^{3}$, Qing Liu ${ }^{3}$, Hao Yang ${ }^{1}$, Jun Ma ${ }^{4}$, Ting-Hua Wang ${ }^{1{ }^{*+}} \mathbb{O}$, Jia Liu ${ }^{1{ }^{*+}}$ and Liu-Lin Xiong ${ }^{1,2,3^{*+}}$

\begin{abstract}
Background: Neonatal hypoxic-ischemic encephalopathy (HIE) represents as a major cause of neonatal morbidity and mortality. However, the underlying molecular mechanisms in brain damage are still not fully elucidated. This study was conducted to determine the specific potential molecular mechanism in the hypoxic-ischemic induced cerebral injury.

Methods: Here, hypoxic-ischemic (HI) animal models were established and primary cortical neurons were subjected to oxygen-glucose deprivation (OGD) to mimic HIE model in vivo and in vitro. The HI-induced neurological injury was evaluated by Zea-longa scores, Triphenyte-trazoliumchloride (TTC) staining the Terminal Deoxynucleotidyl Transferased Utp Nick End Labeling (TUNEL) and immunofluorescent staining. Then the expression of Cytochrome c oxidase subunit 5a (COX5A) was determined by immunohistochemistry, western blotting (WB) and quantitative real time Polymerase Chain Reaction (qRT-PCR) techniques. Moreover, HSV-mediated COX5A over-expression virus was transducted into OGD neurons to explore the role of COX5A in vitro, and the underlying mechanism was predicted by GeneMANIA, then verified by WB and qRT-PCR.

Results: $\mathrm{HI}$ induced a severe neurological dysfunction, brain infarction, and cell apoptosis as well as obvious neuron loss in neonatal rats, in corresponding to the decrease on the expression of COX5A in both sides of the brain. What's more, COX5A over-expression significantly promoted the neuronal survival, reduced the apoptosis rate, and markedly increased the neurites length after OGD. Moreover, Triosephosephate isomerase (TPI) was predicted as physical interactions with COX5A, and COX5A over-expression largely increased the expressional level of TPI.
\end{abstract}

Conclusions: The present findings suggest that COX5A plays an important role in promoting neurological recovery after $\mathrm{HI}$, and this process is related to TPI up-regulation.

Keywords: Neonatal hypoxic-ischemic encephalopathy, COX5A overexpression, Neuronal survival, TPI

*Correspondence: Wangth_email@163.com; liujiaaixuexi@126.com; 499465010@qq.com

†Ya Jiang, Xue Bai, Ting-Ting Li, Ting-Hua Wang, Jia Liu and Liu-Lin Xiong contribute equally to this work

${ }^{1}$ Laboratory Zoology Department, Institute of Neuroscience, Kunming Medical University, Kunming, China

Full list of author information is available at the end of the article

\section{Background}

Neonatal hypoxic-ischemic encephalopathy (HIE) could impact neuronal development and result in abnormal function in whole life, which may underlie the cause of seizures and other severe neurological deficits in children [1]. As a destructive disease that causes the death of infants [2-4], HIE occurs in 1.5/1000 live births in China

(c) The Author(s) 2020. This article is licensed under a Creative Commons Attribution 4.0 International License, which permits use, sharing, adaptation, distribution and reproduction in any medium or format, as long as you give appropriate credit to the original author(s) and the source, provide a link to the Creative Commons licence, and indicate if changes were made. The images or other third party material in this article are included in the article's Creative Commons licence, unless indicated otherwise in a credit line to the material. If material is not included in the article's Creative Commons licence and your intended use is not permitted by statutory regulation or exceeds the permitted use, you will need to obtain permission directly from the copyright holder. To view a copy of this licence, visit http://creativecommons.org/licenses/by/4.0/. The Creative Commons Public Domain Dedication waiver (http://creativecommons.org/publicdomain/zero/1.0/) applies to the data made available in this article, unless otherwise stated in a credit line to the data. 
[5], and $0.2-0.3 \%$ of live births in the United States are recorded with HIE cases. Severely, approximately 25\% of HIE infants die in their first year, while another $25 \%$ survive but with permanent neurological disabilities [6]. The most significant risk factor for HIE is perinatal asphyxia that may occur either in utero or post-natally. Utero asphyxia is caused by inadequate blood flow or oxygen supply resulting in breathing difficulty and even in breathing arrest altogether. Postnatal asphyxia causes neonatal pulmonary failure such as severe hyaline membrane disease, meconium aspiration syndrome, pneumonia as well as congenital heart disease [7-10]. However, brain damage and neuronal cell death through oxidative stress and excitotoxicity [11] are amongst the outcomes of HIE causing high rate of mortality and morbidity in infants.

Increasing treatment studies targeting neonatal HIE have been developed over the years, and currently, hypothermia has been considered as a standard therapy. Neonates receiving hypothermia go through less death and incidence in IQ score below 70, less severe disability as well as attention-executive dysfunction [12]. However, its limited effectiveness remains incompletely certain [13]. Besides the hypothermia, the current therapies including erythropoietin (EPO) [14], hyperbaric oxygen (HBO) $[14]$, Xenon $[15,16]$ and melatonin [17] treatments exert protective effects on the brain and peripheral organs following HIE, but less than satisfactory. The main reason for this phenomenon is that the molecular mechanisms underlying cell damage in HIE still remain vague, and there lacks an effective intervention against development of HIE. Thus novel effective interventions based on the molecular mechanisms are urgently needed.

It has been reported that Cytochrome c oxidase subunit $5 \mathrm{a}(\mathrm{COX} 5 \mathrm{~A})$ combines with COX5b to form the COX5, a subtype of cytochrome c oxidase (COX) related to mitochondria activity [18]. In addition, COX is an endogenous metabolic marker and is closely related to energy metabolism [19]. For example, employing a 4-vessel occlusion model of cerebral ischemia in rats, a previous study revealed the activity of $\mathrm{COX}$ was decreased at $1 \mathrm{~h}$ after ischemia, which may be associated with the neuronal ischemic injury [20]. Moreover, the investigation was expanded in the ischemic-reperfusion model to further explore the effects of mitochondrial protein synthesis and COX activity, and the results showed that the degree of COX activity was also decreased to $90.3 \%$, $80.3 \%, 81.9 \%$ and $83 \%$ of the control values at $15 \mathrm{~min}$ after cerebral ischemia and 1, 3, 24 h after reperfusion, respectively [21]. Likewise, another study reported that COX activity was significantly decreased at $3 \mathrm{~h}$ after traumatic brain injury, and the COX5A subunit was oxdatively modified in the hippocampus [22]. These studies thus indicated that COX5A may play important roles in the process of central nervous system diseases. However, the role of COX5A and its associated mechanism with neonatal HIE remain to be explored.

Here, in order to find out new targets based on COX5A and provide the basic evidence for the treatment of HIE in future, we utilized the animal model of neonatal $\mathrm{HI}$ injury together with primary cortical neurons subjected to oxygen-glucose deprivation (OGD) as in vitro model to analyze the role of COX5A and determine the related molecular mechanisms in HIE insults.

\section{Materials and methods}

Animals and grouping

Forty-seven-day-old postnatal (P7) Sprague-Dawley (SD) male rats (12-15 g) used for in vivo experiments and 21-day-old rats for primary cortical neurons cultures were purchased from the Department of Zoology of Kunming Medical University. Experimental procedures were reviewed following the standard biosecurity and institutional safety procedures and approved by the Ethic Committees at Kunming Medical University in Yunnan province, China (reference number: kmmu2018031). All animals were raised with their mother in plastic cages with soft wood and free access to food and water in a temperature $\left(21-25{ }^{\circ} \mathrm{C}\right.$ ) and humidity (50-60\%)-controlled room. Experimental operations and data analysis can't be done by the same researchers, and data analysis must be dealt by at least two researchers blinded to the experimental design.

\section{Rat model of neonatal HI injury}

In this experiment, we employed the classic Rice-Vannucci method to establish the HI model [23, 24]. Briefly, P7 SD rats were anesthetized with isoflurane (4\% for induction, $2 \%$ for sustained inhalation anesthesia) after they were weighed and numbered. The hypoxic chamber was set at $37{ }^{\circ} \mathrm{C}$ with humidity $50-80 \%$ before the operation. Briefly, the midline of the ventral cervical skin was cut followed by a blunt dissection of parenchyma, to expose the right common carotid. Subsequently, the right common carotid was ligated by an electric coagulator. Then, the wound was stitched and animals were returned to their mother for $1 \mathrm{~h}$ before being kept in a hypoxic chamber under $8 \% \mathrm{O}_{2}$ and $92 \% \mathrm{~N}_{2}$ (flow rate $3 \mathrm{~L} / \mathrm{min}$ ) for $2 \mathrm{~h}$. Rats in the sham group underwent the same procedures, apart from ligation of the right carotid artery.

\section{Zea-longa score}

Zea-longa scores were used to evaluate the neurological function in neonatal rats subjected to HI injury, verifying the successful modeling. All rats underwent the zealonga score test to evaluate the neurological dysfunction 
at $0 \mathrm{~h}, 2 \mathrm{~h}, 4 \mathrm{~h}, 8 \mathrm{~h}$, and $16 \mathrm{~h}$ after HI. The specific scoring criteria were as follows: 0 points-no signs of nerve injury; 1 point-the contralateral forepaw lost ability to become fully stretched; 2 points-animals turns to one side while walking; 3 points-walking is unstable, falling to one side; 4 points-loss of consciousness [25].

\section{Tissue acquisition}

For morphological analysis, rats in the sham $(n=5$ for TTC staining, $\mathrm{n}=3$ for immunohistochemical and immunofluorescent staining) and HI ( $\mathrm{n}=5$ for TTC staining, $\mathrm{n}=3$ for immunohistochemical and immunofluorescent staining) groups were euthanatized at $16 \mathrm{~h}$ after $\mathrm{HI}$ under deep anesthesia with $4 \%$ isoflurane (sustained inhalation anesthesia) for $2 \mathrm{~min}$. Then, after the perfusion of $0.9 \%$ normal saline followed by $4 \%$ paraformaldehyde, the brain was harvested and put into $4 \%$ paraformaldehyde for more than $72 \mathrm{~h}$. With paraffin embedded, the brain Sects. $(5 \mu \mathrm{m})$ were prepared for immunohistochemical staining and immunofluorescent staining, and the core infarction regions were the key observations.

For molecular biology analysis, rats in the sham $(n=6$ for qRT-PCR and $n=6$ for WB) and HI ( $n=6$ for qRTPCR and $n=6$ for WB) groups were sacrificed at $16 \mathrm{~h}$ after the surgery under deep anesthesia by continuously inhaling $4 \%$ isoflurane for 2 min followed by perfusion with $0.9 \%$ normal saline. Thereafter, the white infarcted area and non-infracted area of the contralat-eral hemisphere were removed and stored at $-80^{\circ} \mathrm{C}$ for further WB and qRT-PCR.

\section{Triphenyte-trazoliumchloride (TTC) staining}

To evaluate the brain damages after HI, TTC staining was performed to observe the infarction of brain tissues in neonatal $\mathrm{HI}$ rats. The whole brain from the sham and $\mathrm{HI}$ rats was quickly removed at $16 \mathrm{~h}$ after rats being deeply anaesthetized with $4 \%$ isoflurane, brain tissues was taken out (operating on ice) and frozen in a refrigerator at $-20{ }^{\circ} \mathrm{C}$ for $10 \mathrm{~min}$ before being cut into five pieces ( $2 \mathrm{~mm}$ each). Afterwards, the sections were placed in $1 \%$ TTC solution at $37^{\circ} \mathrm{C}$ and incubated for $30 \mathrm{~min}$. The sections were then captured using the digital camera. The non-ischemic necrotic area was pale red, and the ischemic necrotic tissue was white. The ratio of infarct was analyzed using Image J software [26].

\section{Immunohistochemistry in vivo}

The immunohistochemistry was employed to detect COX5A expression. The collected brain was paraffinembedded and cut into $5 \mu \mathrm{m}$ sections, then fixed on the glass slides. Before immunohistochemical staining, the sections were deparaffinized and a circle was drawn around the tissue with a PAP pen. Thereafter, sections were washed for three times with $0.01 \mathrm{M}$ PBS buffer $(\mathrm{PH}=7.45), 5 \mathrm{~min}$ each. Next, $3 \%$ hydrogen peroxide was added onto the sections followed by incubating at $37{ }^{\circ} \mathrm{C}$ for $15 \mathrm{~min}$ and washed as described above. In order to block the non-specific binding in the tissue, a drop of $5 \%$ goat serum (Solarbio, S9070) was added on the section and incubated at $37{ }^{\circ} \mathrm{C}$ for $30 \mathrm{~min}$. Subsequently, the primary antibodies (COX5A, Zhongshanjinqiao, rabbit, 1:50) were added and $2 \%$ goat serum was used as the negative control, lefting overnight in a refrigerator at $4{ }^{\circ} \mathrm{C}$. The next day, sections were washed with 0.01 M PBS buffer for three times, followed by addition of enhancement solution, incubation at $37^{\circ} \mathrm{C}$ for $20 \mathrm{~min}$ and washing with $0.01 \mathrm{M}$ PBS three times. Subsequently, the secondary antibody (Zhongshanjinqiao, goat antirabbit IgG) was added and the sections were incubated at $37{ }^{\circ} \mathrm{C}$ for $1 \mathrm{~h}$, and then washed with PBS. Next, DAB staining was performed for $5 \mathrm{~min}$ following the manufacturer's protocol. To perform nuclei staining, the sections were treated with hematoxylin solution for $5 \mathrm{~min}$, and soaked in $1 \% \mathrm{HCl}$-ethanol solution for $10 \mathrm{~s}$ followed by $5 \%$ ammonia treatment for $1 \mathrm{~min}$. Afterwards, they were dried with serial dilutions of ethanol $(75 \%, 80 \%$, $85 \%, 95 \%$, and $100 \%$ ) and treated with xylene solution for $2 \mathrm{~min}$, finally sealed with neutral resins. The immunepositive pictures were captured using a light microscope (Leica DMI 6000 B inverted microscope, Germany). For quantification, three sections of each brain with $50 \mu \mathrm{m}$ apart were selected, and five fields of each slice were randomly collected at $100 \times$. The positive cells (dark brown labeled) in each field were quantified via Image-Pro Plus 6.0 software (MediaCybernetics, Silver Spring, MD, USA).

\section{Primary cortical neuron cultures and COX5A-over-expression HSV vector transduction}

COX5A overexpression transduction was conducted for the further functional verification of COX5A's role in the process of $\mathrm{HI}$. The primary cortical neurons were isolated and cultured from freshly dissected brains of rat pups at postnatal day one. In brief, the cortical tissues were dissociated to disperse the cells in a neuro-basal medium (Gibco, California-USA) supplemented with $2 \%$ B27 and $0.5 \mathrm{mM}$ of glutamine. The cells were plated on poly-L-lysine and laminin coated vessels at the density as described above and were incubated at $37^{\circ} \mathrm{C}$ with $5 \% \mathrm{CO}_{2}$. The media were then replaced on alternating days as described before [27]. Thereafter, a low toxicity HSV-COX5A virus was purchased from Sky Bio (BeijingChina) to explore the function of COX5A according to the manufacturer's protocol. Briefly, HSV-COX5A virus was transducted into the neurons after 5 days of incubation at a MOI $=10 . \mathrm{GFP}$, emitting green fluorescence, 
expressed from a co-infection with a GFP-expressing virus in neurons after transduction. Then the infection rate was calculated (GFP -positive cells/total neurons). Detection of the green fluorescence and qRT-PCR were employed to demonstrate the successful transduction and over-expression of COX5A.

\section{Oxygen-glucose deprivation (OGD)}

After culturing for 7 days, OGD was established to mimic the HI condition in vitro. In detail, following three washes with PBS, the neurons were incubated using glucose-free medium (Gibco, USA) in an anaerobic chamber containing $5 \% \mathrm{CO}_{2}$ and $95 \% \mathrm{~N}_{2}$ at $37{ }^{\circ} \mathrm{C}$ for $90 \mathrm{~min}$ as described earlier [27]. Subsequently, cells were returned to original medium, then placed in a normoxic chamber with $95 \%$ air and $5 \% \mathrm{CO}_{2}$ for $16 \mathrm{~h}$ before further testing.

\section{Immunofluorescent staining in vivo and in vitro}

In order to examine the neuronal viability after $\mathrm{HI}$ injury in vivo and in vitro, immunofluorescence staining was carried out. In vivo, the brain slices $(\mathrm{n}=3$ brains, and three sections of each brain with $50 \mu \mathrm{m}$ apart) were subsequently deparaffinized and washed three times with $0.01 \mathrm{M} \mathrm{PBS}$ and pre-incubated for $30 \mathrm{~min}$ with $0.3 \%$ TritonX-100 in 5\% normal goat serum. Then they were incubated overnight at $4{ }^{\circ} \mathrm{C}$ with primary antibodies against NeuN (mouse, Bioss, 1:100). The next day, the primary antibodies were removed and sections were rinsed with $0.01 \mathrm{M}$ PBS buffer for three times before being incubated with the fluorescence-labeled secondary antibodies of Alexa 488 (anti-mouse, Invitrogen, 1:100) for $1 \mathrm{~h}$ at $37{ }^{\circ} \mathrm{C}$. Counterstaining of the nuclei was performed using DAPI staining. As NeuN was located on the cytoplasm and nucleus of neurons, so the positive staining was the nuclei of neurons labeled with green fluorescence and co-stained with DAPI, which showed blue fluorescence. Finally, the slices were observed under a fluorescent microscope (Leica, CM1860, Germany) at $200 \times$. In detail, five fields of each slice were randomly selected for the estimation of relative number of NeuN positive cells (green fluorescence labeled) using Image-Pro Plus 6.0 software.

In vitro, to study the expression of COX5A in OGD condition and the effect of COX5A on Neuronal Class III $\beta$-Tubulin (Tuj $1^{+}$) in neurons, the primary neuronal cells from the normal, OGD, OGD $+\mathrm{NC}$, OGD + COX5A groups were cultured on glass cover slips. In brief, the cell sections ( $n=6$ in each group) were fixed with $4 \%$ formalin and blocked with $0.3 \%$ TritonX-100 in 5\% normal goat serum for $30 \mathrm{~min}$ at $37^{\circ} \mathrm{C}$. The slips were then incubated with anti-COX5A (Mouse, Santa Cruz, 1:100) and anti-beta III Tubulin antibody (Mouse, abcam, 1:200) respectively at $37^{\circ} \mathrm{C}$ for $16-18 \mathrm{~h}$. Then, the cells were rinsed three times with PBS before being incubated with fluorescence-labeled secondary antibodies of Dylight 594 (anti-mouse, abbkine, 1:200) at $37^{\circ} \mathrm{C}$ for $1 \mathrm{~h}$. Then DAPI was used to counterstain the nuclei. Five fields in each well were randomly collected at $400 \times$ with a fluorescence microscope (Leica, CM1860, Germany). The COX5A positive cells and average cell number of $\mathrm{Tuj}^{+}$cells, average length of the neurite (about calculating 15 neurons) were quantified by Image-Pro Plus 6.0 software.

\section{Terminal deoxynucleotidyl transferased Utp nick end labeling (Tunel) in vivo and in vitro}

Tunel staining was performed to determine the apoptotic occurrence in the cortical infarction region of neonatal $\mathrm{HI}$ rats and cultured primary cortical neurons subjected to OGD. Briefly, the brain slices $(\mathrm{n}=3$ brains, and three sections of each brain with $50 \mu \mathrm{m}$ apart) and cell sections ( $\mathrm{n}=6$ in each group) were rinsed three times with $0.01 \mathrm{M}$ PBS buffer for $5 \mathrm{~min}$, and $50 \mu \mathrm{l}$ of $0.01 \%$ sodium citrate and $0.1 \%$ TritonX-100 was added onto each slice and incubated for $30 \mathrm{~min}$ at $37^{\circ} \mathrm{C}$. Then, the slices were incubated with the tunel mixture reagent (tunel label solution: Tune enzyme solution $=9: 1$ ) for $1 \mathrm{~h}$ at $37{ }^{\circ} \mathrm{C}$. For the negative control, only the tunel label solution was added dropwise. After that, counterstaining of the nuclei was performed using DAPI staining. Finally, tunel positive cells were labeled by red fluorescence and can be co-stained with DAPI staining, which showed blue fluorescence. For quantification, five fields on each slice around the core infarction regions were randomly chosen for imaging with a fluorescence microscope (Leica, CM1860, Germany) at $200 \times$. Then, about 200 cells in each field were quantified by Image-Pro Plus 6.0 software. Then, apoptosis rate was calculated as (tunel positive cells/DAPI cells) $\%$.

\section{Quantitative real-time polymerase chain reaction (qRT-PCR)}

QRT-PCR was performed to detect the mRNA expression of COX5A and other candidate genes after COX5A over-expression. The primers were designed using Premier 5.0 software, then verified via the BLAST software and synthesised by Takara Bio Inc (Takara, Japan). Total RNAs from the fresh cortex and cultured neurons $(n=6$ per group) were extracted using the RNAiso plus kit (TaKaRa, JAPAN) and cDNA Synthesis was performed using the ReviertAid Kit (Thermo Fisher Scientific Inc.). The levels of Gstp1, Sod2, Rho-GDIa, TPI, COX5A and $\beta$-actin mRNA expression in the samples were estimated by qRT-PCR (CFX-96, Bio-Rad, USA) according to the manufacturer's instructions using the primers described in Table 1. Briefly, each reaction was performed in a volume of $20 \mu \mathrm{l}$ mixture consisting of $10 \mu \mathrm{l}$ of SYBR Green 
Table 1 The premier sequences

\begin{tabular}{|c|c|c|c|}
\hline Factor & Forward & Reverse & $\begin{array}{l}\text { Annealing } \\
\text { temperature } \\
\left({ }^{\circ} \mathrm{C}\right)\end{array}$ \\
\hline$\beta$-actin & 5'GAAGATCAAGATCATTGCTCC $3^{\prime}$ & 5'TACTCCTGCTTGCTGATCCA3' & 52 \\
\hline $\operatorname{cox} 5 \mathrm{~A}$ & 5'GTTGGACCAATCATAGGCGCT3' & 5'CAATGTCGATCACATGCACCA3' & 56 \\
\hline Rho-GDla & 5'TACAAGCCTCCAGCCCAGAA 3' & 5' AAGGTCAAGCGGGTCACAAT3' & 53 \\
\hline Gstp1 & $5^{\prime}$ TCAAGGCTCGCTCAAGTCCA 3' & 5' TTGCATCGAAGGTCCTCCAC 3' & 52 \\
\hline Sod2 & 5' CCCTGACCTGCCTTACGACT 3' & 5' AGCGACCTTGCTCCTTATTG 3' & 53 \\
\hline TPI & 5' GGGGCAACTGGAAGATGAA 3' & $5^{\prime}$ CCTGGCGAAGTCGATGTAG 3' & 52 \\
\hline
\end{tabular}

Table 2 The antibody information of WB

\begin{tabular}{llll}
\hline Protein & Manufacturer & Lot number & Dilution ratio \\
\hline COX5A (primary antibody) & Santa cruz & sc-376907 & $1: 500$ \\
$\beta$-actin (primary antibody) & Bioss & bsm-33139M & $1: 2000$ \\
TPI (primary antibody) & Bioss & bs-4042R & $1: 300$ \\
HRP, Goat Anti-Mouse IgG (secondary antibody) & Abbkine & A21020 & $1: 500$ \\
HRP, Goat Anti-Mouse IgG (secondary antibody) & Abbkine & A21010 & $1: 500$ \\
\hline
\end{tabular}

master mix, $1 \mu \mathrm{l}$ of cDNA, $7.8 \mu \mathrm{l}$ of water, and $0.6 \mu \mathrm{l}$ each of forward and reverse primers. Finally, the data were analyzed using a comparative critical threshold (Ct) method where relative expression was calculated as $2^{-\Delta \Delta C t}$ method.

\section{Western blotting (WB)}

WB was employed with the intent to detect the protein expression of COX5A in the cortex of neonatal HI rats and of TPI in OGD neurons after COX5A overexpression. The right and left cortex from rats of the sham and HI group and primary neuronal cells from the normal, OGD, OGD + NC, OGD + COX5A groups ( $n=6$ wells in each group) were collected and lysed with RIPA buffer containing a proteinase inhibitor cocktail (Roche) on ice for $30 \mathrm{~min}$, then the lysates were centrifuged at $12,000 \mathrm{rpm}$ for $10 \mathrm{~min}$ at $4{ }^{\circ} \mathrm{C}$. Protein concentrations were determined by BCA protein quantification kit. The protein samples were boiled and denatured with a loading buffer and $40 \mu \mathrm{g}$ of protein was added to each well and run at $120 \mathrm{~V}$ for $90 \mathrm{~min}$. After that, the proteins in the gel were transferred to a PVDF membrane at a current of $200 \mathrm{~mA}$. The PVDF membrane was then blocked with 5\% skim milk powder for $90 \mathrm{~min}$ at room temperature, and further incubated with related primary antibodies (seen in Table 2) at $4{ }^{\circ} \mathrm{C}$ overnight. $\beta$-actin was used as an internal control. Thereafter, membranes were incubated with the secondary antibodies for $2 \mathrm{~h}$ (seen in Table 2). Finally, the membranes were developed and the bands were visualized with the ECL (ECL Western blotting kit) luminescence solution. The quantitative analysis was carried out by Image J software. The data were expressed as a ratio of the protein of interest band to $\beta$-actin band optical density values.

\section{Bioinformatics prediction}

GeneMANIA provides many large, publicly available biological datasets to find related genes. These include protein-protein, protein-DNA and genetic interactions, pathways, reactions, gene and protein expression data, protein domains and phenotypic screening profiles. Data is regularly updated. The relationship between Gstp1, Sod2, Rho-GDIa, TPI and COX5A was predicted using the GeneMANIA website (http:// genemania.org/).

\section{Statistical analysis}

Data were expressed as mean \pm standard deviation (SD). The comparison between two groups was performed using a Student's t test. For comparison of multiple groups in vitro experiments, ANOVA with least-significant difference (LSD) or Dunnett's T3 post hoc test was applied, if equal variances were found, LSD was performed; otherwise, Dunnett's T3 was used [28]. All statistical analyses were performed with SPSS18.0 software (IBM Corporation, NY, USA). $p<0.05$ was considered statistically significant. 


\section{Results}

$\mathrm{HI}$ induced cerebral infarction and cell apoptosis

TTC staining and zea-longa scoring were used to evaluate the reliability of the HI model establishment in this study. The zea-longa test showed an obvious increase in the score of rats in the HI group compared with the sham group (HI vs. sham, $p<0.05$, Fig. 1a). TTC staining showed that the infarcted brain tissue was white without detectable staining, while the unaffected brain tissue was stained red. Compared with the sham group, a quantitative analysis showed that the infarct volume in HI group was obviously higher than that in the sham group (HI vs. sham, $p<0.01$, Fig. 1b, c), which further confirmed the HI model was established successfully. In addition, the TUNEL staining revealed that the apoptosis rate in the infraction region of the right cortex in the HI group was significantly increased in comparison with the sham group (HI vs. sham, $p<0.01$, Fig. 1d, e).

\section{HI caused neuron loss}

Immunofluorescent staining showed that the nuclei of neurons were labeled with NeuN. The fluorescence intensity weakened and positive cells of NEUN specific for neurons decreased in the HI group, in comparison to sham group (HI vs. sham, $p<0.05$, Fig. 2a, b).

\section{COX5A expression was decreased after $\mathrm{HI}$ injury in vivo and in vitro}

To detect the expression changes of COX5A in the cerebral cortex after $\mathrm{HI}$, we performed immunohistochemistry, qRT-PCR and WB. The immunohistochemistry results showed that COX5A was localized in the nucleus of the cortex at $16 \mathrm{~h}$ (Fig. 3a, b). Compared with the sham group, the positive cells of COX5A were significantly reduced in the left and right cortex in the $\mathrm{HI}$ group (HI vs. sham, $p<0.01$, Fig. 3c, d). COX5A protein and mRNA expression were significantly diminished in the left and right cortex as compared with the sham group revealed by WB (HI vs. sham, $p<0.01$, Fig. 3e, f) and qRT-PCR detection (HI vs. sham, $p<0.05$, Fig. $3 g$, h).

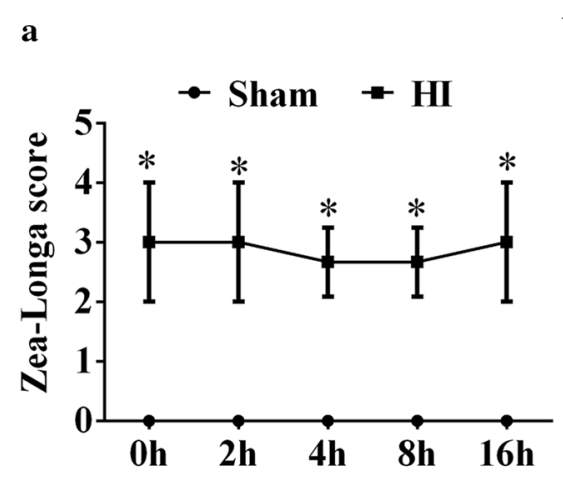

b

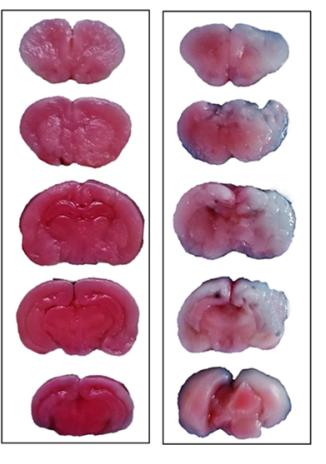

c

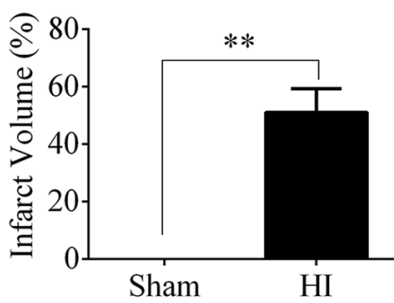

d

DAPI

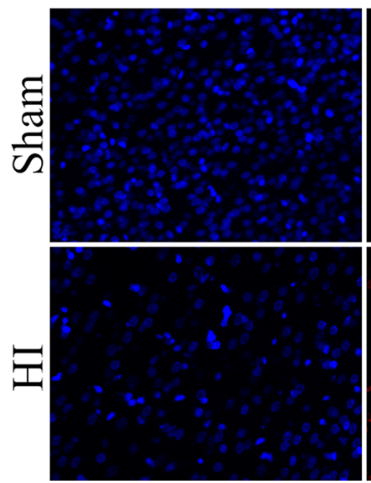

Tunel
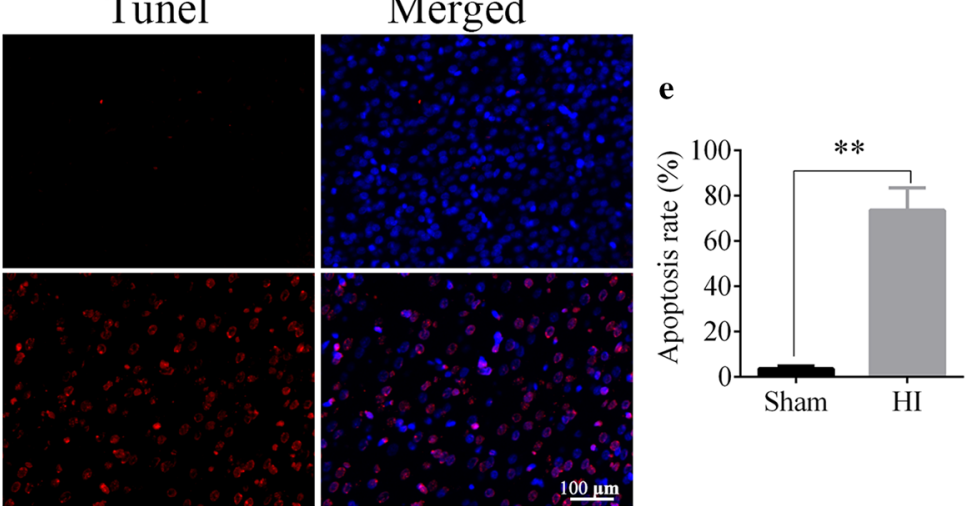

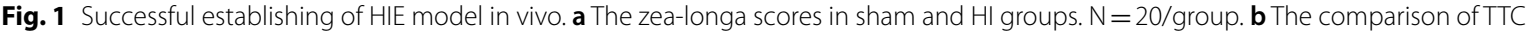
staining between sham and $\mathrm{HI}$ groups, and the white staining represented the ischemic area. $\mathrm{N}=5 /$ group. $\mathbf{c}$ The percentage of infarct area in sham and HI groups. d Tunel assay showed the apoptotic cells in right cortex. Red fluorescence represents the positive tunel staining, and blue is the nucleus staining with DAPI. $N=3$ /group. e Apoptosis rate in the right cortex, revealed by the tunel/DAPI. HI hypoxic-ischemic, $h$ hour, TTC Triphenyte-trazoliumchloride, tunel terminal deoxynucleotidyl transferasedUtp nick end labeling staining. Data are presented as the mean \pm SD. ${ }^{*} p<0.05,{ }^{* *} p<0.01$. Scale bar $=100 \mu \mathrm{m}$ 


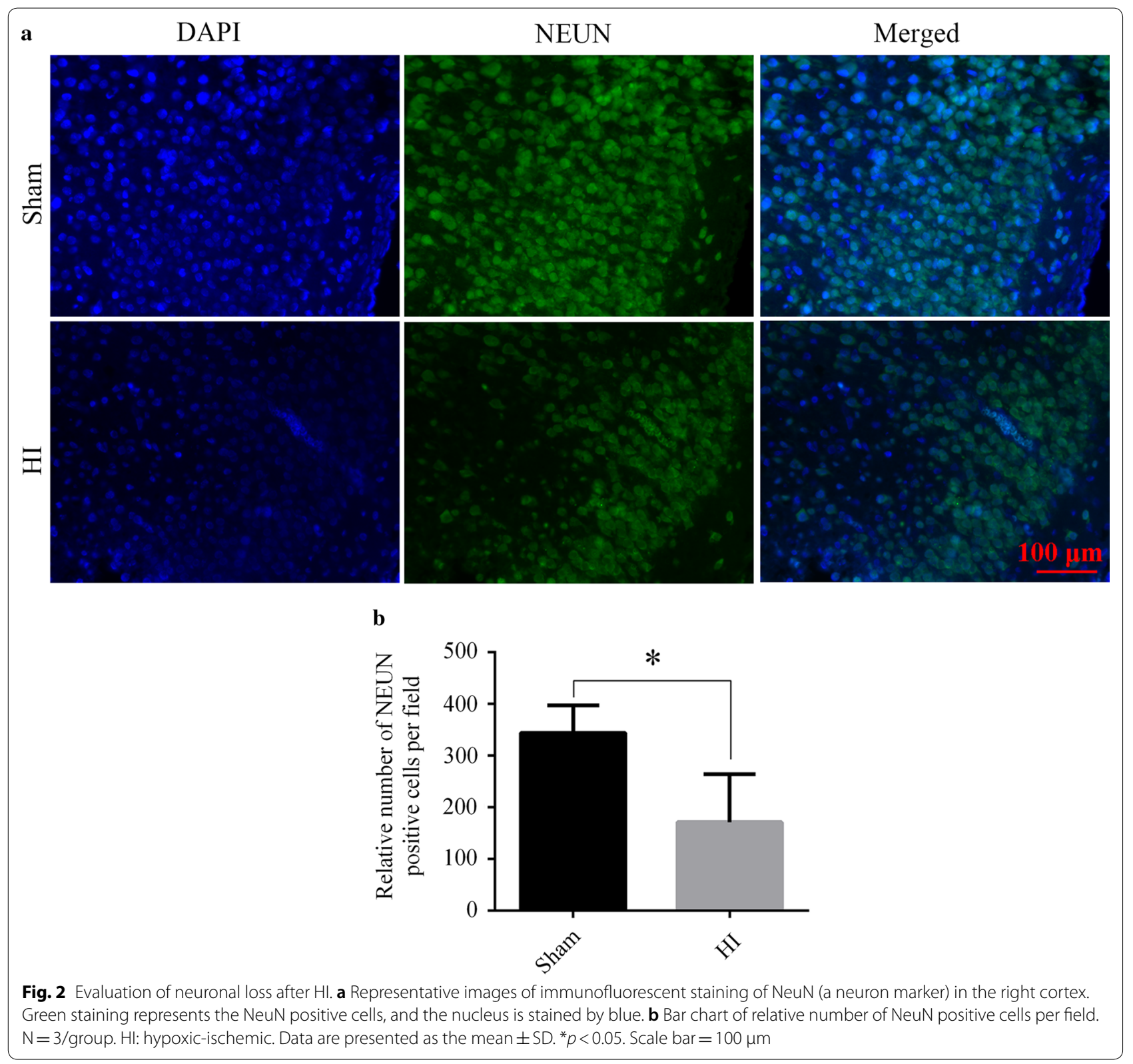

In the bright-filed images, we observed that compared with the normal group, differentiation and development of neurons was slower in the OGD group 90 min after OGD operation (Fig. 4a). In addition, COX5A mRNA expression was also decreased in the OGD cells (OGD vs. normal, $p<0.05$, Fig. 4 b). Moreover, immunofluorescent staining further revealed that the number of COX5A positive cells was decreased significantly in OGD group as compared with the Normal group (OGD vs. normal, $p<0.01$, Fig. $4 \mathrm{c}, \mathrm{d}$ ).

\section{HSV-COX5A vector was successfully transducted} in the primary neuronal cells

The successful transduction of HSV-COX5A vector into the primary cortical neurons was demonstrated by the green fluorescence emitted by GFP in Fig. 5a, and the infection rate reached about $92 \%$. What's more, qRTPCR showed that the mRNA expression of COX5A was significantly increased in the COX5A over-expression group in both normal and OGD conditions as compared with the NC (COX5A-ORF vs. NC, $p<0.01$, Fig. 5b, c). 


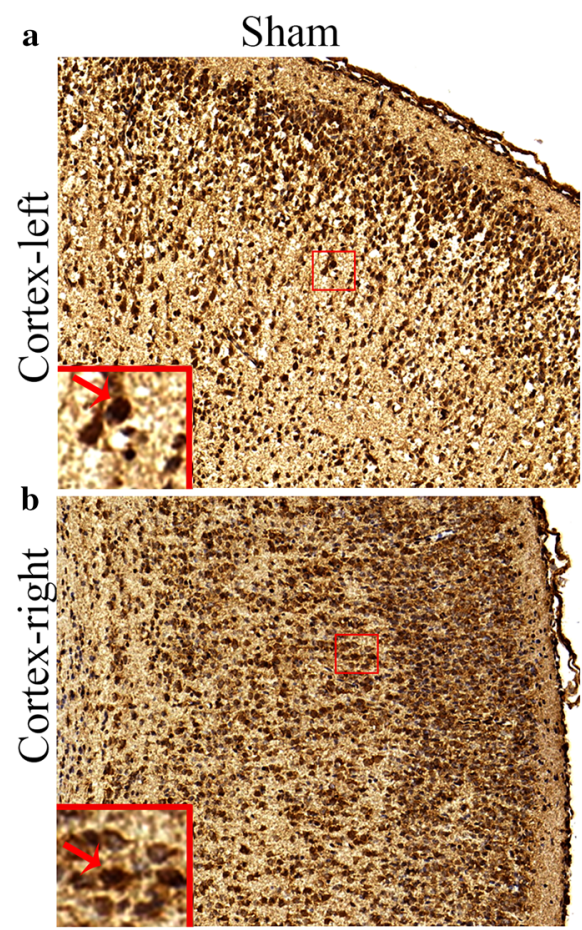

e
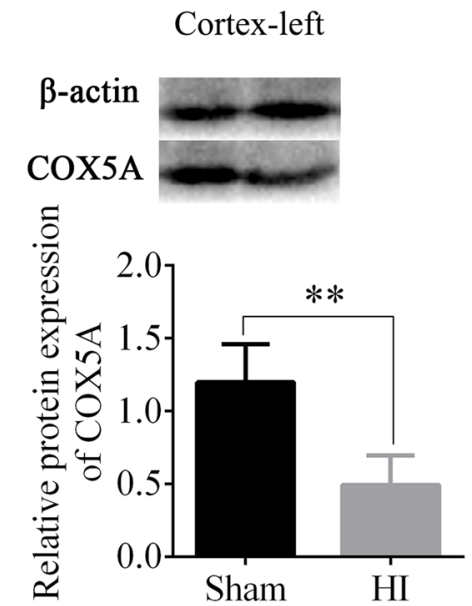

f

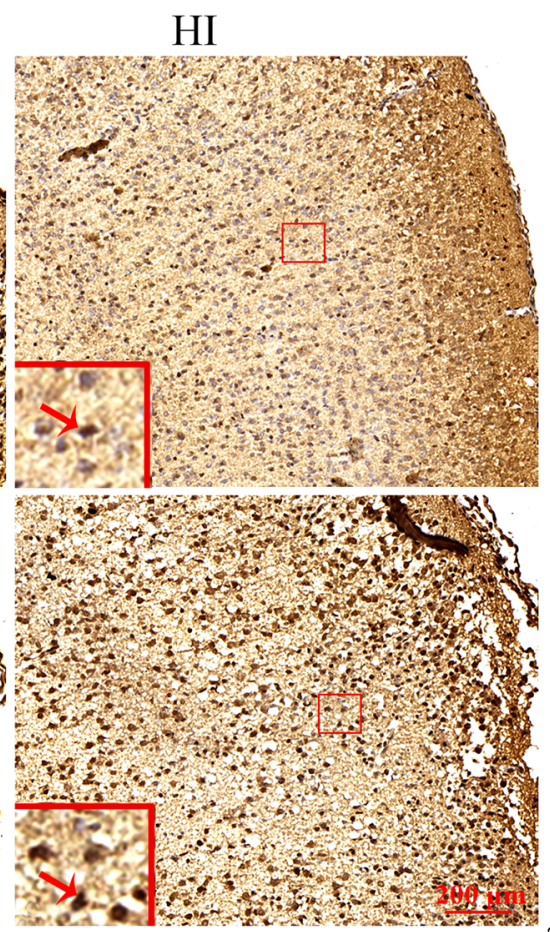

c

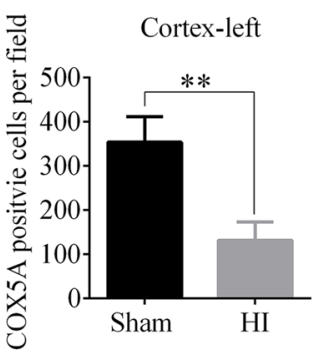

d
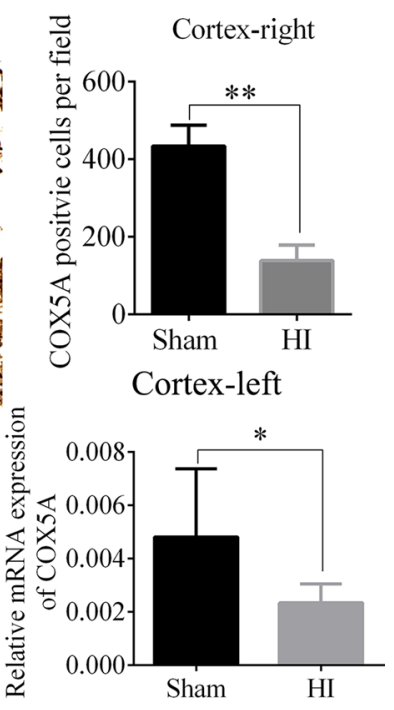

Cortex-right

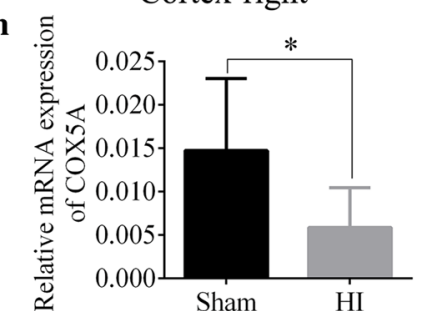

Fig. 3 The expression of COX5A after HI. a, b Representative images of immunohistochemical staining of COX5A in left- and right-cortex, Red arrows represent the COX5A positive cells, which were stained by the dark brown colour. $\mathrm{N}=3 / \mathrm{group}$. $\mathbf{c}$, $\mathbf{d}$ Bar charts of the COX5A positive cells in left- and right- cortex. $\mathrm{N}=3$ /group. e, $\mathbf{f} W B$ results of the COX5A protein expression in left- and right-cortex. $\mathrm{N}=6 / \mathrm{group}$. $\mathbf{g}$, $\mathbf{h}$ qRT-PCR results of the COX5A mRNA expression in left- and right-cortex. $\mathrm{N}=6$ /group. HI hypoxic-ischemic, COX5A cytochrome c oxidase subunit 5a, WB western blotting, $q R T-P C R$ quantitative real-time polymerase chain reaction. Data are presented as the mean $\pm \mathrm{SD} .{ }^{*} p<0.05,{ }^{* *} p<0.01 . \mathrm{Scale}$ bar $=200 \mu \mathrm{m}$

It demonstrated that COX5A was successfully overexpressed in the cortical neurons.

\section{Over-expression of COX5A promoted the neuronal outgrowth and decreased the cell apoptosis}

To study the influence of COX5A overexpression on neurites outgrowth and neuronal apoptosis, immunofluorescent staining of Tuj1 and TUNEL assay were employed
(Fig. 6a). Quantitative evaluation revealed that the OGD treated cells had overall shorter length of neurites and less cell number relative to normal group (OGD vs. normal, $p<0.01$, Fig. 6a, d, e). In addition, OGD also led to increased cell apoptosis indicated by the elevated apoptosis rate (OGD vs. normal, $p<0.01$, Fig. 6b, f). However, COX5A over-expression in OGD neurons showed a significant elevation in the cell number, and increase in the 

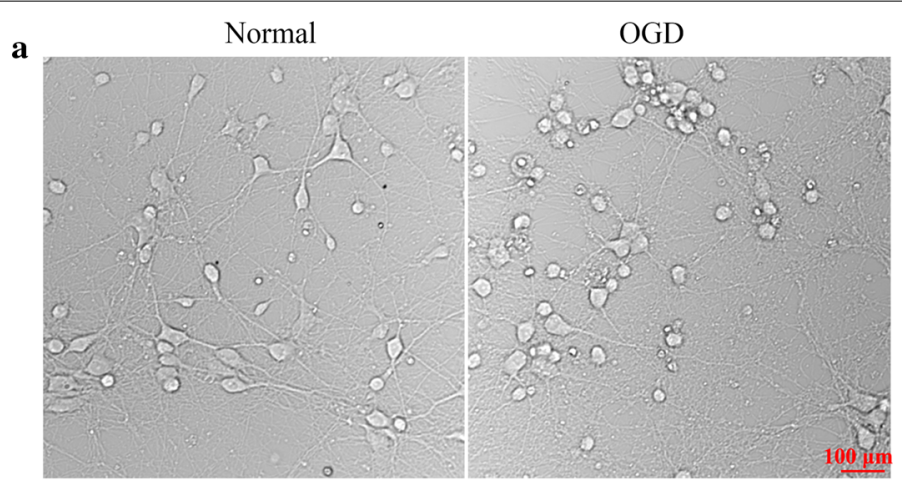

b

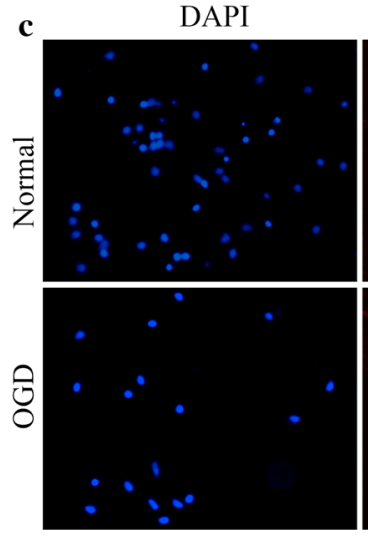

$\operatorname{COX} 5 \mathrm{~A}$
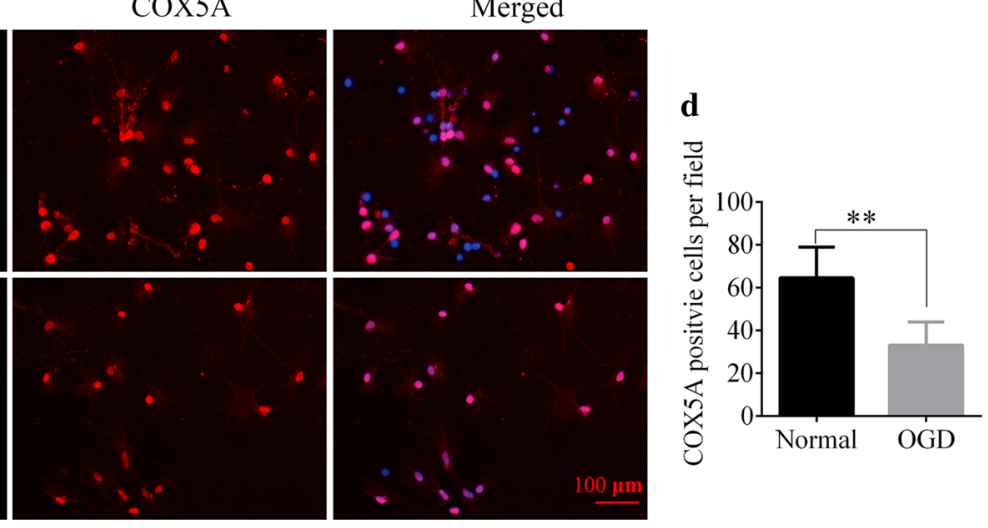

Fig. 4 The expression of COX5A in OGD neurons. a The bright field of neurons in normal and OGD groups. Scale bar = $100 \mu \mathrm{m}$. b Quantitative analysis of relative mRNA expression of COX5A in neuron. $\mathrm{N}=6$ /group. $\mathbf{c}$ Immunofluorescent staining of COX5A in normal and OGD groups. Red staining represents the COX5A positive cells, and the nucleus is stained by blue, $\mathrm{n}=3 / \mathrm{group}$. Scale bar $=200 \mu \mathrm{m}$. $\mathbf{d}$ The COX5A positive cells per field were calculated. $N=6 /$ group. OGD oxygen-glucose-deprivation, COX5A cytochrome c oxidase subunit 5a, qRT-PCR quantitative real-time polymerase chain reaction. Data are presented as the mean $\pm S D .{ }^{*} p<0.05,{ }^{* *} p<0.01$

neurite lengths as well as decrease in apoptosis rate in comparison with the OGD $+\mathrm{NC}$ group (OGD $+\mathrm{COX} 5 \mathrm{~A}$ vs. OGD $+\mathrm{NC}, p<0.05, p<0.01$, Fig. 6d-f). These revealed COX5A over-expression could maintain the neurites and inhibit neuronal apoptosis so as to improve neurological function.

\section{COX5A over-expression markedly up-regulated TPI expression}

GeneMANIA was used to explore the underlying molecular mechanism of COX5A over-expression in regulating neurites outgrowth and cell apoptosis in the cortical neurons. Interestingly, four genes (Gstp1, TPI, Rho GDIa and Sod2) were screened out. What's more, it was found that Gstp1 and TPI are co-localized with COX5A, and the TPI was physically interacted with COX5A (Fig. 7a). Moreover, the qRT-PCR verification showed that as compared to the normal group, the mRNA levels of Rho-GDIa and Gstp1 were markedly increased while the expression of TPI was reduced in OGD group, and Sod2 levels didn't respond to the OGD (OGD vs. normal, $p<0.01$, Fig. $7 \mathrm{~b}-$ e). Conversely, in the OGD cells over-expressing COX5A, the mRNA expression of Rho-GDIa and Sod2 was significantly reduced (OGD $+\mathrm{COX} 5 \mathrm{~A}$ vs. $\mathrm{OGD}+\mathrm{NC}$, $p<0.05$, Fig. $7 \mathrm{~b}, \mathrm{e})$, while the Gstp1 and TPI expression was significantly increased (OGD +COX5A vs. $\mathrm{OGD}+\mathrm{NC}, p<0.01$, Fig. $7 \mathrm{c}, \mathrm{d})$. Altogether, the findings revealed that only TPI expression was positively correlated with COX5A. WB analysis further confirmed the protein expression of TPI was increased after COX5A overexpression in OGD neurons (OGD+COX5A vs. $\mathrm{OGD}+\mathrm{NC}, p<0.05$, Fig. $7 \mathrm{f})$.

\section{Discussion}

In this study, employing a rat model with neonatal hypoxic-ischemic in vivo, and OGD neuronal cell injury model in vitro, we found that the expression of COX5A was significantly decreased after $\mathrm{HI}$ with more neuronal damages and apoptosis in the right cortical injury area. In addition, over-expression of COX5A effectively promoted the outgrowth of neuronal neurite and reduced apoptosis in neurons subjected to OGD, and the potential molecular mechanisms are closely related to the 

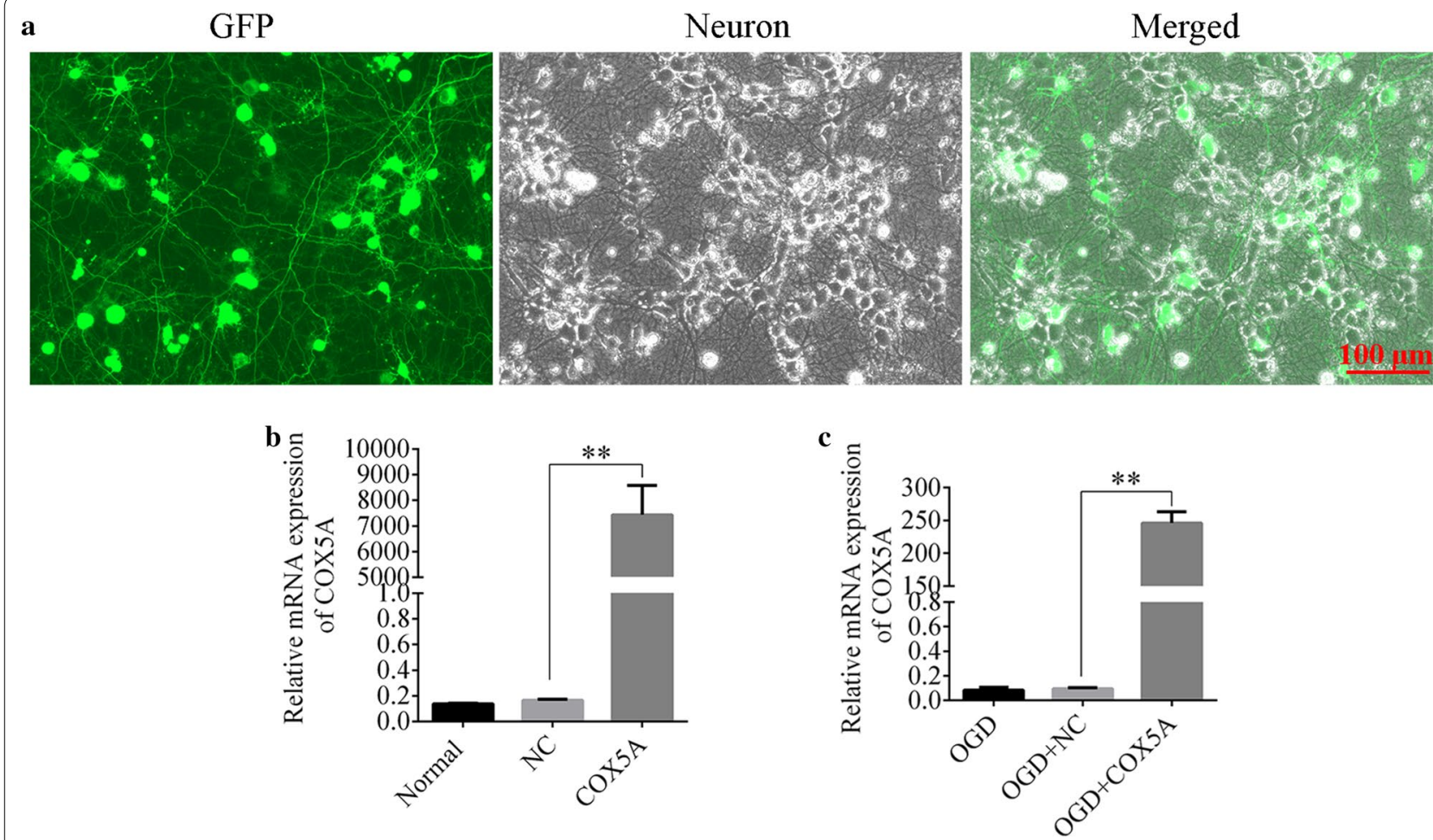

Fig. 5 Transduction and verification of HSV contained COX5A over-expression. a The effective transduction of the HSV virus into neurons at $\mathrm{MOI}=10$. Green fluorecence corresponded to a green fluorescent protein which was a marker protein in HSV vector. Gray corresponded to neuron in the bright field with PH channel. $\mathrm{N}=6$ /group. $\mathbf{b}$, c Verification of COX5A expression using qRT-PCR in normal and OGD conditions, respectively. $\mathrm{N}=6$ /group. OGD oxygen-glucose-deprivation, NC negative control, GFP green fluorescent protein, COX5A-ORF cytochrome coxidase subunit 5a over-expression, Data are presented as the mean \pm SD. ${ }^{* *} p<0.01$. Scale bar $=100 \mu \mathrm{m}$

up-regulation of TPI expression. This may provide a new idea for future clinical treatment with $\mathrm{HI}$ injury.

\section{The $\mathrm{HI}$ model was successfully established in $\mathrm{P7}$ rats}

In this study, the neonatal HI model was successfully established based on the classic Rice-Vannucci method of neonatal HI [23, 24]. Previously, an MRI study compared the HI model by Rice-Vannucci and the neonatal stroke filament occlusion, which revealed that the neonatal stroke injury is restricted in the middle cerebral artery, while it spreads collaterally in the Rice-Vannucci HI model [29] Therefore, the Rice-Vannucci model of neonatal $\mathrm{HI}$ has been used the most in the basic study. The zea-longa scores were used to evaluate the neurological function in ischemic model, and also applied to verify the hypoxic-ischemic model establishment in neonatal rats $[30,31]$, Moreover, literature proved that the brain injury of P7 rats equals that of full-term or near-term human fetuses [32]. Additionally, P7 rats represent the peak brain growth, which occurs at term humans and is equivalent to 34 weeks' gestation [33]. Therefore, $\mathrm{HI}$ model in the present study was established in P7 neonatal rats by the right common carotid artery ligation and subsequent hypoxia for $2 \mathrm{~h}$. As a result, the cerebral injuries were mainly concentrated in the right side of the brain [24], thus, we focused on the right cerebral hemisphere in the later observation of brain damage.

\section{Decreased expression of COX5A induced the neuronal injury}

In the present study, the expression of COX5A was decreased after HI injury. Multiple studies [34-37] reported that the expression of COX5A decreased in a variety of central nervous system diseases, which in turn caused an imbalance in neuronal energy regulation. Moreover, Wei HL reported that down-regulation of COX5A seriously impaired the sensory function in a neuroplastic model of SD rat after dorsal root ganglion resection [38]. What's more, the down-regulation of COX5A led to mitochondrial damage and dysfunction, further accelerated disease progression in the course of HIE disease [39, 40]. COX5A reduction has also been demonstrated close correlation with acute myocardial infarction [41] and diabetes [42]. However, little information has been reported about the role of COX5A in neuronal growth after SCI injury. Exactly in this study, 


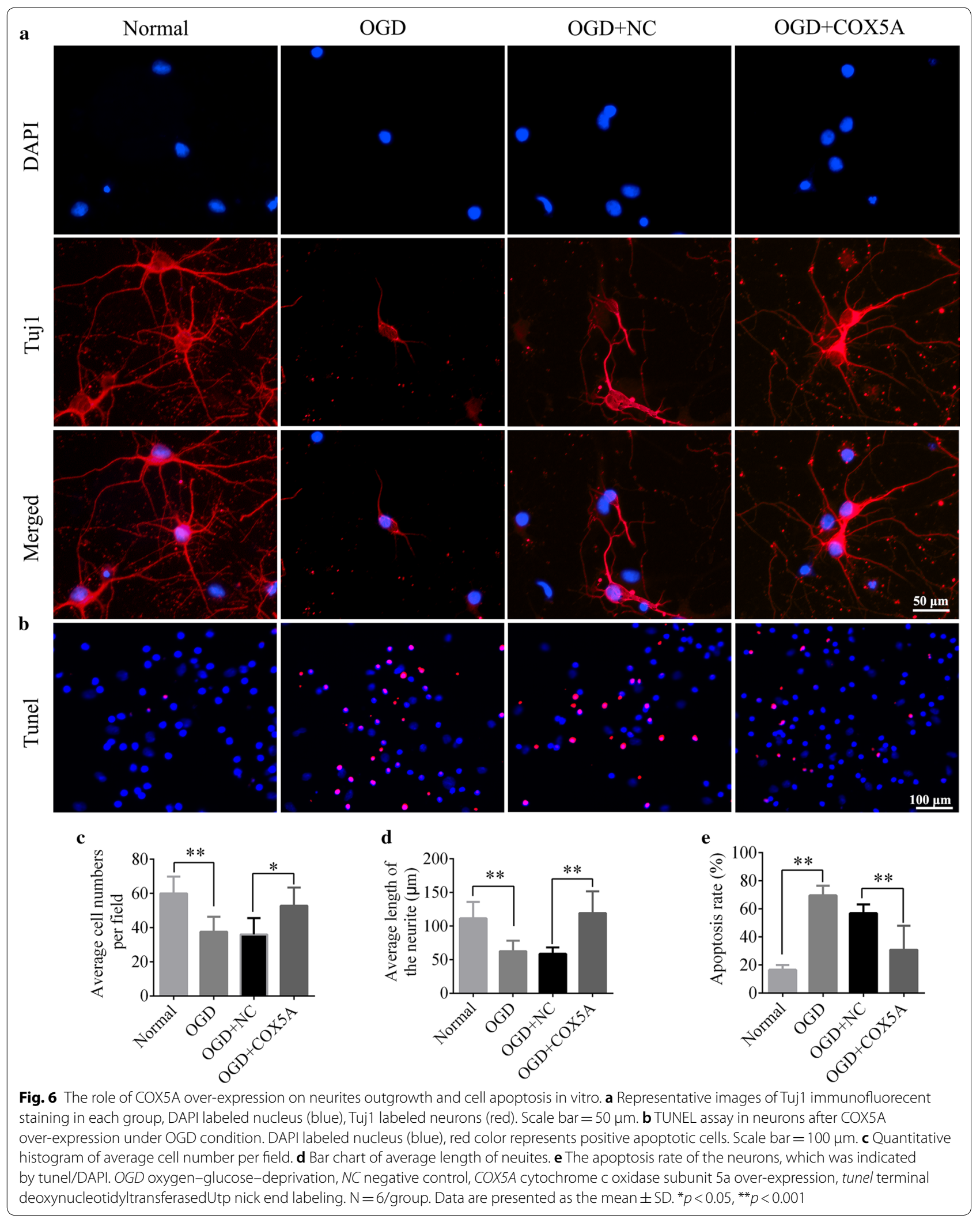




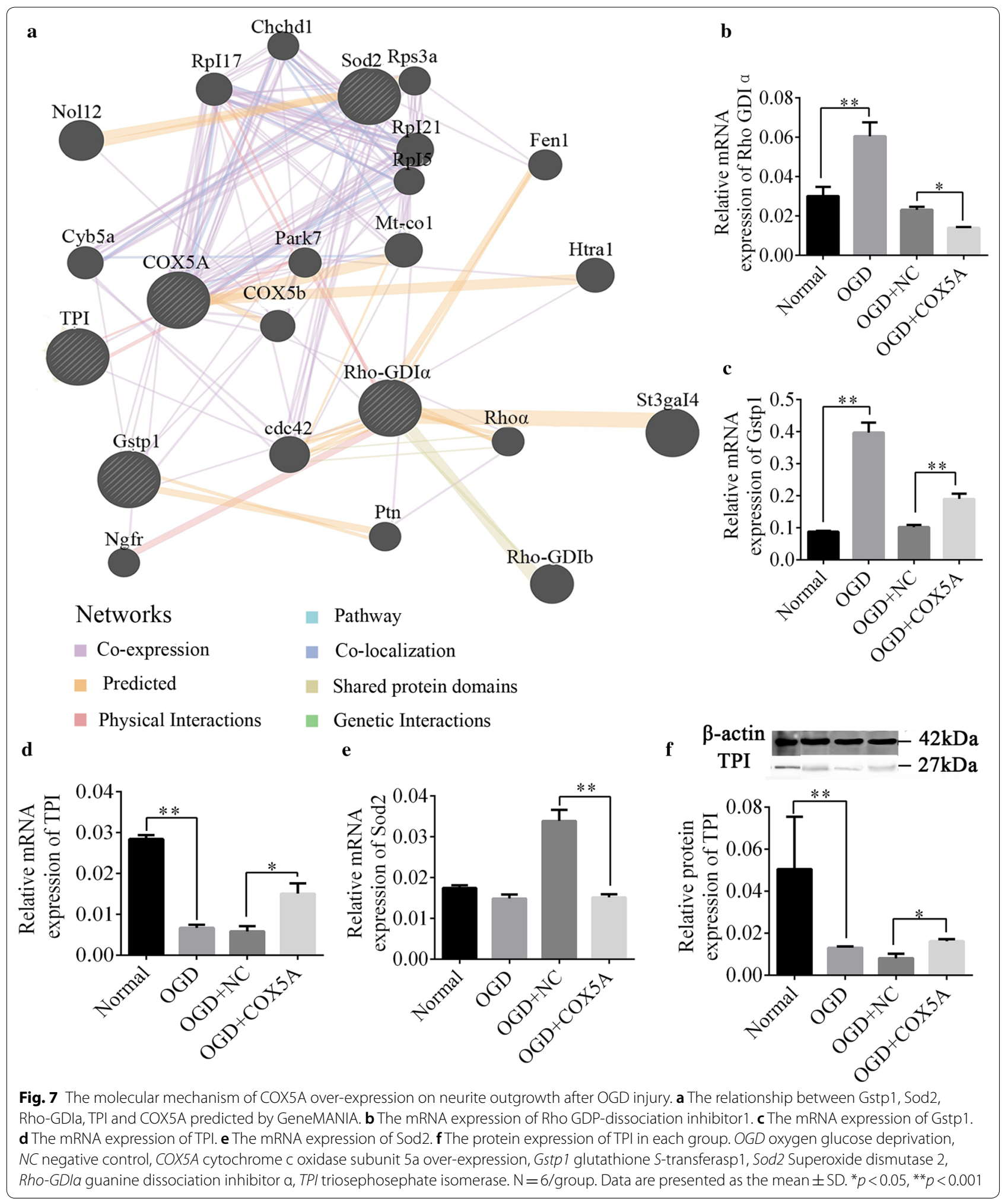

we demonstrated the cell number reached about 60 per field and the each neurite length exceeded $100 \mu \mathrm{m}$ under normal condition, which were conversely reduced a lot in
OGD neurons. Nevertheless, the decreased trend was significantly reversed in OGD neurons when overexpressing COX5A. These indicated the decrease of COX5A may 
induce the neuronal damage and cell apoptosis, while overexpression of COX5A could promote the neurites length and depressed neuronal apoptosis, strengthening the crucial role of COX5A in the process of HIE.

\section{Over-expressing COX5A for neuronal improvement is associated with TPI up-regulation}

Our study found that the over-expression of COX5A promoted the outgrowth of neurites and reduced apoptosis in neurons subjected to OGD, therefore uncovering its crucial role in the recovery of HIE. As for the role of COX5A, a previous study found that the COX5A expression was up-regulated in the injured area after acupuncture treatment with rats after spinal cord injury. Besides, XiYang YB constructed COX5A over-expression transgenic mice and found that hippocampal neurons have enlarged cell bodies and increased dendritic complexity, and hippocampal-dependent spatial learning and memory ability got improved [43]. These indicated that increasing COX5A expression may exert neuroprotective effect on the central nervous system diseases. Similarly, our study found that the expression of COX5A was reduced in HIE rats and OGD neurons, and transduction of HSV-COX5A over-expressing virus into cortical neurons in vitro reduced apoptosis and increased neuronal neurite lengths to alleviate the OGD injury, therefore supporting the positive role of COX5A in neural repair after HI. For the mechanism of COX5A, it was wellestablished that COX5A over-expression could increase CGRP densities in lamina of the spinal cord [44] to further promote wound healing. Whereas, the present study concealed that the over-expression of COX5A could markedly up-regulate the TPI level in OGD neurons, which suggests TPI may be the downstream targets of COX5A in the HI process. As a glycolytic enzyme [45], mutations of TPI in coding gene revealed its association with neurological phenotypes [46]. Also, TPI deficiency is associated with progressive neurological dysfunction and commonly leads to the death of the carrier in early years of childhood [47]. Recently, through development of genetically engineered variants of TPI, it was hypothesized that this enzyme has non-catalytic function that is important for the survival of neurons, which explains the neurological complications associated with TPI deficiency [48]. Nevertheless, TPI is identified as a target for arginine methyltransferase 5 and cyclin dependent kinase $2[49,50]$. These findings demonstrate the strict regulation of TPI by cell cycle pathways, and explain their neurological importance. In fact, new evidence showed the firm relationship between cell cycle imbalance and neurological disorder [51], in which, Alzheimer's disease is considered one example where failure in cell balancing results in massive neurodegeneration [52]. Together, TPI up-regulation in this study could be useful to improve neurological function in the HIE insults, and its role is positively correlated with COX5A.

\section{Conclusion}

Collectively, we demonstrated the decrease of COX5A in the cortex after HI and OGD injury, whereas, overexpressing COX5A in OGD condition could largely improve cell function. The possible mechanism is associated with TPI up-regulation. These findings may contribute to the mechanism explanation of HIE and provide new idea for the treatment of HIE in future clinic trial.

\section{Abbreviations \\ OGD: Oxygen-glucose-deprivation; NC: Negative control; COX5A: Cytochrome c oxidase subunit 5a over-expression; Gstp1: Glutathione S-transferasp1; Sod2: Superoxide dismutase 2, Rho-GDla: Guanine dissociation inhibitor a; TPI:Triosephosephate isomerase; TUNEL: Terminal Deoxynucle- otidyl TransferasedUtp Nick End Labeling; TTC: Triphenyte-trazoliumchloride; HI: Hypoxic-ischemic; WB: Western blotting; qRT-PCR: Quantitative real-time polymerase chain reaction; GFP: Green Fluorescent Protein.}

\section{Acknowledgements \\ None.}

\section{Authors' contributions}

All authors are responsible for the accuracy of data in this study, and approved the final version of the manuscript. $Y J, J L$ and $L L X$ designed and supervised the whole study; $X B$ and TTL performed all the experiments; JM, LYL, and MA contributed the newly added experiments in the revised stage; $Y J, Y Z, Y H, Y Z$, $Q L$, and $H Y$ participated in the part of experiments and data analysis. $X B, J L$, LLX and THW revised the final paper. All authors read and approved the final manuscript.

\section{Funding}

This study was supported by the Key Grant of biological Medicine from Yunnan Province (No. 2018ZF007-4), and the Program Innovative Research Team in Science and Technology in Yunnan Province (No. 2017HC007), and Yunnan Applied Basic Research Project-Union foundation (No. 2017FE467). They paid for the experimental materials and for the expenses incurred in the process of data collection and analysis.

\section{Availability of data and materials}

The datasets analyzed during the current study available from the corresponding author on reasonable request.

\section{Ethics approval and consent to participate}

Experimental procedures were reviewed following the standard biosecurity and institutional safety procedures and approved by the Ethic Committees at Kunming Medical University in Yunnan province, China (reference number: kmmu2018031).

\section{Consent for publication}

Not applicable.

\section{Competing interests}

The authors confirm that there is no conflict of interest in this article.

\section{Author details}

${ }^{1}$ Laboratory Zoology Department, Institute of Neuroscience, Kunming Medical University, Kunming, China. ${ }^{2}$ School of Pharmacy and Medical Sciences, Division of Health Sciences, University of South Australia, Adelaide 5000, South Australia. ${ }^{3}$ National Traditional Chinese Medicine Clinical Research Base and Western Medicine Translational Medicine Research Center, Department of Cardiac and Cerebral Diseases, Affiliated Traditional Chinese Medicine 
Hospital, Southwest Medical University, Luzhou 646000, China. ${ }^{4}$ Department of Neurosurgery, The First People's Hospital of Zhaotong, Zhaotong 657000, China.

Received: 6 December 2019 Accepted: 17 April 2020 Published online: 29 April 2020

\section{References}

1. Dixon BJ, et al. Neuroprotective strategies after neonatal hypoxic ischemic encephalopathy. Int J Mol Sci. 2015;16(9):22368-401.

2. Doycheva $\mathrm{D}$, et al. Granulocyte-colony stimulating factor in combination with stem cell factor confers greater neuroprotection after hypoxicischemic brain damage in the neonatal rats than a solitary treatment. Transl Stroke Res. 2013;4(2):171-8.

3. Shetty J. Neonatal seizures in hypoxic-ischaemic encephalopathyrisks and benefits of anticonvulsant therapy. Dev Med Child Neurol. 2015;57(Suppl 3):40-3.

4. Volpe JJ. Perinatal brain injury: from pathogenesis to neuroprotection. Ment Retard Dev Disabil Res Rev. 2001;7(1):56-64.

5. Kurinczuk JJ, White-Koning M, Badawi N. Epidemiology of neonatal encephalopathy and hypoxic-ischaemic encephalopathy. Early Hum Dev. 2010;86(6):329-38.

6. Logitharajah P, Rutherford MA, Cowan FM. Hypoxic-ischemic encephalopathy in preterm infants: antecedent factors, brain imaging, and outcome. Pediatr Res. 2009;66(2):222-9.

7. Barkovich AJ, et al. Proton spectroscopy and diffusion imaging on the first day of life after perinatal asphyxia: preliminary report. Am J Neuroradiol. 2001;22(9):1786-94

8. Bruce DA, Schut L. Spinal lipomas in infancy and childhood. Childs Brain. 1979;5(3):192-203.

9. Ferreira GC, McKenna MC. L-Carnitine and acetyl-L-carnitine roles and neuroprotection in developing brain. Neurochem Res. 2017:42(6):1661-75.

10. Zarifi MK, et al. Prediction of adverse outcome with cerebral lactate level and apparent diffusion coefficient in infants with perinatal asphyxia. Radiology. 2002;225(3):859-70.

11. Northington FJ, Chavez-Valdez R, Martin LJ. Neuronal cell death in neonatal hypoxia-ischemia. Ann Neurol. 2011;69(5):743-58.

12. Shankaran S. Therapeutic hypothermia for neonatal encephalopathy. Curr Treat Options Neurol. 2012;14(6):608-19.

13. Laptook $A R$, et al. Effect of therapeutic hypothermia initiated after 6 hours of age on death or disability among newborns with hypoxic-ischemic encephalopathy: a randomized clinical trial. JAMA. 2017;318(16):1550-60.

14. Wei $L$, et al. Hyperbaric oxygenation promotes neural stem cell proliferation and protects the learning and memory ability in neonatal hypoxicischemic brain damage. Int J Clin Exp Pathol. 2015;8(2):1752-9.

15. Schmidt $M$, et al. Xenon attenuates cerebral damage after ischemia in pigs. Anesthesiology. 2005;102(5):929-36.

16. Dingley J, et al. Xenon provides short-term neuroprotection in neonatal rats when administered after hypoxia-ischemia. Stroke. 2006;37(2):501-6.

17. Yildiz EP, Ekici B, Tatli B. Neonatal hypoxic ischemic encephalopathy: an update on disease pathogenesis and treatment. Expert Rev Neurother. 2017;17(5):449-59.

18. Allen $L A$, et al. Isoforms of yeast cytochrome c oxidase subunit $V$ affect the binuclear reaction center and alter the kinetics of interaction with the isoforms of yeast cytochrome c. J Biol Chem. 1995;270(1):110-8.

19. Perez-Gracia E, Torrejon-Escribano B, Ferrer I. Dystrophic neurites of senile plaques in Alzheimer's disease are deficient in cytochrome c oxidase. Acta Neuropathol. 2008;116(3):261-8.

20. Racay P, et al. Mitochondrial calcium transport and mitochondrial dysfunction after global brain ischemia in rat hippocampus. Neurochem Res. 2009;34(8):1469-78.

21. Racay $P$, et al. Ischemia-reperfusion induces inhibition of mitochondrial protein synthesis and cytochrome c oxidase activity in rat hippocampus. Physiol Res. 2009;58(1):127-38.
22. Opii WO, et al. Proteomic identification of oxidized mitochondrial proteins following experimental traumatic brain injury. J Neurotrauma. 2007;24(5):772-89.

23. Rice JE, Vannucci RC, Brierley JB. The influence of immaturity on hypoxicischemic brain damage in the rat. Ann Neurol. 1981;9(2):131-41.

24. Chen W, et al. Matrix metalloproteinases inhibition provides neuroprotection against hypoxia-ischemia in the developing brain. J Neurochem. 2009;111(3):726-36

25. Carter RJ, Morton J, Dunnett SB. Motor coordination and balance in rodents. Curr Protoc Neurosci. 2001;15(1):8-12.

26. Rousselet E, Kriz J, Seidah NG. Mouse model of intraluminal MCAO: cerebral infarct evaluation by cresyl violet staining. J Vis Exp. 2012;69:e4038.

27. Liu XH, et al. Hyperbaric oxygenation reduces long-term brain injury and ameliorates behavioral function by suppression of apoptosis in a rat model of neonatal hypoxia-ischemia. Neurochem Int. 2013;62(7):922-30.

28. Kim HY. Statistical notes for clinical researchers: Sample size calculation 3. Comparison of several means using one-way ANOVA. Restor Dent Endod. 2016:41(3):231-4

29. Ashwal $\mathrm{S}$, et al. Comparison of two neonatal ischemic injury models using magnetic resonance imaging. Pediatr Res. 2007;61(1):9-14.

30. Zhang ZB, et al. MiRNA-7a-2-3p inhibits neuronal apoptosis in oxygenglucose deprivation (OGD) model. Front Neurosci. 2019;23(13):16.

31. Xiong LL, et al. Suppression of PDGF induces neuronal apoptosis after neonatal cerebral hypoxia and ischemia by inhibiting P-PI3K and P-AKT signaling pathways. Brain Res. 2019;15(1719):77-88.

32. Clancy B, Darlington RB, Finlay BL. Translating developmental time across mammalian species. Neuroscience. 2001;105(1):7-17.

33. Bennet $L$, et al. Cell therapy for neonatal hypoxia-ischemia and cerebral palsy. Ann Neurol. 2012;71(5):589-600.

34. Antequera $D$, et al. Cytoplasmic gelsolin increases mitochondrial activity and reduces Abeta burden in a mouse model of Alzheimer's disease. Neurobiol Dis. 2009;36(1):42-50.

35. Callahan LA, Supinski GS. Downregulation of diaphragm electron transport chain and glycolytic enzyme gene expression in sepsis. J Appl Physiol. 2005;99(3):1120-6.

36. Liang HL, Ongwijitwat S, Wong-Riley MT. Bigenomic functional regulation of all 13 cytochrome c oxidase subunit transcripts in rat neurons in vitro and in vivo. Neuroscience. 2006;140(1):177-90.

37. Ongwijitwat S, Wong-Riley MT. Is nuclear respiratory factor 2 a master transcriptional coordinator for all ten nuclear-encoded cytochrome c oxidase subunits in neurons? Gene. 2005;360(1):65-77.

38. Wei $\mathrm{H}$. Role of COX5A in neuroplasticity following partial dorsal root rhizotomy and electro-acupuncture. Kunming: Kunming Medical University; 2012.

39. Zhu C, et al. Involvement of apoptosis-inducing factor in neuronal death after hypoxia-ischemia in the neonatal rat brain. J Neurochem. 2003;86(2):306-17.

40. Modjtahedi N, et al. Apoptosis-inducing factor: vital and lethal. Trends Cell Biol. 2006;16(5):264-72

41. Hui J, Chun Y, Da Z. Effects of Chinese herbs capable of replenishing qi, nourishing yin and activating blood circulation and their compatibility on differentially expressed genes of ischemic myocardium. Chin Sci Bull. 2009:54(18):3278-82

42. LuY, et al. Predict the neurological recovery under hypothermia after cardiac arrest using C0 complexity measure of EEG signals. Conf Proc IEEE Eng Med Biol Soc. 2008;2008:2133-6.

43. XiYang $Y B$, et al. Sodium channel voltage-gated beta 2 plays a vital role in brain aging associated with synaptic plasticity and expression of COX5A and FGF-2. Mol Neurobiol. 2016;53(2):955-67.

44. Fukuda R, et al. HIF-1 regulates cytochrome oxidase subunits to optimize efficiency of respiration in hypoxic cells. Cell. 2007;129(1):111-22.

45. Albery WJ, Knowles JR. Free-energy profile of the reaction catalyzed by triosephosphate isomerase. Biochemistry. 1976;15(25):5627-31.

46. Tanaka KR, Zerez CR. Red cell enzymopathies of the glycolytic pathway. Semin Hematol. 1990;27(2):165-85.

47. Orosz F, Olah J, Ovadi J. Triosephosphate isomerase deficiency: facts and doubts. IUBMB Life. 2006;58(12):703-15.

48. Roland BP, et al. Evidence of a triosephosphate isomerase non-catalytic function crucial to behavior and longevity. J Cell Sci. 2013;126(Pt 14):3151-8. 
49. Kim C, et al. Regulation of post-translational protein arginine methylation during HeLa cell cycle. Biochim Biophys Acta. 2010;1800(9):977-85.

50. Lee $\mathrm{WH}$, et al. Functional inactivation of triosephosphate isomerase through phosphorylation during etoposide-induced apoptosis in HeLa cells: potential role of Cdk2. Toxicology. 2010;278(2):224-8.

51. Khurana V, Feany MB. Connecting cell-cycle activation to neurodegeneration in Drosophila. Biochim Biophys Acta. 2007;1772(4):446-56.
52. Yang Y, Geldmacher DS, Herrup K. DNA replication precedes neuronal cell death in Alzheimer's disease. J Neurosci. 2001;21 (8):2661-8.

\section{Publisher's Note}

Springer Nature remains neutral with regard to jurisdictional claims in published maps and institutional affiliations.
Ready to submit your research? Choose BMC and benefit from:

- fast, convenient online submission

- thorough peer review by experienced researchers in your field

- rapid publication on acceptance

- support for research data, including large and complex data types

- gold Open Access which fosters wider collaboration and increased citations

- maximum visibility for your research: over $100 \mathrm{M}$ website views per year

At BMC, research is always in progress.

Learn more biomedcentral.com/submissions 